\title{
Internato rural médico como importante estratégia de (trans)formação da educação médica: um relato de experiência sobre o trabalho interdisciplinar no controle da hipertensão arterial
}

Medical internship as an important strategy of (trans)formation of medical education: an account of experience on interdisciplinary work in the control of arterial hypertension

Trabajo práctico rural médico como importante estrategia de (trans)formación de la educación médica: un relato de experiencia sobre el trabajo interdisciplinario en el control de la hipertensión arterial

Rodrigo Mitre Cotta ${ }^{1}$; Isadora Brandão Pelucio ${ }^{2}$; Luciana Saraiva da Silva ${ }^{3}$; Fernanda Mitre Cotta ${ }^{4}$; Filipe Souza Fernandes ${ }^{5}$; Robson Costa Freitas ${ }^{6}$; Rosângela Minardi Mitre Cotta $^{7}$

\section{Resumo}

A evidência científica aponta para potenciais vantagens educacionais da inserção dos estudantes de Medicina em cenários de Atenção Primária à Saúde (APS). Relativo à hipertensão arterial (HA), 60 a $80 \%$ dos casos podem ser tratados em nível de APS, pois são de fácil diagnóstico, não requerem tecnologia sofisticada, podendo ser controlados com medicamentos de baixo custo e mudanças de estilo de vida. $\mathrm{O}$ objetivo foi apresentar o relato de experiência da atuação de acadêmicos de Medicina em trabalho interprofissional no controle da HA. A investigação baseou-se em três etapas complementares: estudo observacional a fim de conhecer o território e as necessidades locais, palestras dialogadas para os portadores de HA e coleta dos dados em consultas individualizadas. Participaram do estudo 15 portadores de HA, sendo a maioria mulheres; idosos, com média de idade de 63 anos; com prevalência de $60 \%$ de sobrepeso e média de índice de massa corporal (IMC) de $27,1 \mathrm{~kg} / \mathrm{m} 2$. Encontraram-se falhas no uso de medicamentos anti-hipertensivos, devido à falta de acompanhamento periódico pelos profissionais. Após a realização de intervenções, 86,7\% tiveram redução nos valores da pressão. Os achados apontam para a importância de um acompanhamento sistemático, contínuo e longitudinal dos portadores de HA, e a importância do internato médico em

\footnotetext{
${ }^{1}$ Médico pela Faculdade de Medicina de Barbacena (FUNJOBE), Barbacena, Minas Gerais. Residente de Ortopedia e Traumatologia no Hospital Santa Casa de Misericórdia de Belo Horizonte. Avenida P.H. Rolfs, s/n, Campus Universitário, Viçosa, Minas Gerais, Brasil, CEP 36571-000. Email: rodrigomcotta@ hotmail.com

${ }^{2}$ Médica pela Faculdade de Medicina de Barbacena (FUNJOBE), Barbacena, Minas Gerais, Brasil. Residente de Clínica Médica no Hospital Santa Casa de Misericórdia de São Sebastião do Paraíso. Avenida P.H. Rolfs, s/n, Campus Universitário, Viçosa, Minas Gerais, Brasil, CEP 36571-000. Email: isa_bpelucio_br@yahoo.com.br

${ }^{3}$ Mestre em Ciência da Nutrição pela Universidade Federal de Viçosa (UFV). Doutoranda do Programa de PósGraduação em Ciência da Nutrição da UFV. Avenida P.H. Rolfs, s/n, Campus Universitário, Viçosa, Minas Gerais, Brasil, CEP 36571-000. E-mail: lucianassnut@gmail.com

${ }^{4}$ Médica pela Faculdade de Medicina de Barbacena (FUNJOBE), Barbacena, Minas Gerais, Brasil. Residente de Ginecologia e Obstetrícia no Hospital Sofia Feldman. Avenida P.H. Rolfs, s/n, Campus Universitário, Viçosa, Minas Gerais, Brasil, CEP 36571-000. Email: fernandamitrec@ hotmail.com

${ }^{5}$ Médico pela Faculdade de Medicina de Barbacena (FUNJOBE), Barbacena, Minas Gerais. Residente de Psiquiatria no Centro Hospitalar Psiquiátrico de Barbacena (FHEMIG). Avenida P.H. Rolfs, s/n, Campus Universitário, Viçosa, Minas Gerais, Brasil, CEP 36571-000. Email: filipefernandes777@ hotmail.com

${ }^{6}$ Enfermeiro da Estratégia de Saúde da Família de Catas Altas da Noruega, Minas Gerais, Brasil. Avenida P.H. Rolfs, s/n, Campus Universitário, Viçosa, Minas Gerais, Brasil, CEP 36571-000. Email: robson.400@ gmail.com

7 Doutora em Saúde Pública pela Universidad de Valencia, Espanha. Professora Associada do Departamento de Nutrição e Saúde da Universidade Federal de Viçosa (UFV). Avenida P.H. Rolfs, s/n, Campus Universitário, Viçosa, Minas Gerais, Brasil, CEP 36571-000. Email: rosangelaminardi@ hotmail.com
} 
ISSN 2179-6750

serviços de APS, visando à capacitação dos futuros médicos e a experiência de trabalho interprofissional. O estudo demonstra ainda a importância da APS, porta de entrada dos indivíduos nos serviços de saúde, e o cenário onde a prevenção de complicações deve ser estabelecida.

Descritores: Educação Médica; Educação em Saúde, Hipertensão; Atenção Primária à Saúde.

\section{Abstract}

Scientific evidence points to potential educational benefits of integration of medical students in Primary Health Care (PHC). For arterial hypertension (AH), $60-80 \%$ of cases can be treated at the level of PHC, as they are easy to diagnose, do not require sophisticated technology and can be controlled with low cost and lifestyle changes medications. The aim was to present the report of the experience of medical students acting in interprofessional work in controlling hypertension. The research was based on three complementary stages: observational study in order to know the territory and local needs, dialogued lectures for $\mathrm{AH}$ patientes and data collection in individual queries. The study enrolled 15 patients with hypertension, most women; elderly, with a mean age of 63 years; with $60 \%$ prevalence of overweight and mean body mass index (BMI) of $27.1 \mathrm{~kg} / \mathrm{m}^{2}$. They have found flaws in the use of antihypertensive drugs, due to lack of regular monitoring by professionals. After conducting interventions, $86.7 \%$ had reduction in pressure values. The findings point to the importance of systematic monitoring, continuous and longitudinal of $\mathrm{AH}$, and the importance of medical internship in PHC services, aimed at training future doctors and interprofisional work experience. The study also demonstrates the importance of PHC, gateway of individuals in health services, and the scene where the prevention of complications should be established.

Key-words: Medical Education; Health Education; Hypertension; Primary Health Care.

\section{Resumen}

La evidencia científica apunta para las ventajas educativas de la integración de los estudiantes de Medicina en centros de Atención Primaria de Salud (APS). Sobre la presión arterial (HA), el 60$80 \%$ de los casos se puede tratar en el nivel de APS, ya que son fáciles de diagnosticar, no requieren tecnología sofisticada, puede ser controlado con medicamentos de bajo costo y cambios de estilo de vida. El objetivo fue presentar un relato de experiencia de los estudiantes de Medicina que actúan en el trabajo interprofesional en el control de la HA. La investigación se basó en tres etapas complementarias: estudio observacional, conferencias dialogadas para los portadores de HA y recolección de datos por medio de consultas individuales. 15 pacientes participaron en el estudio, la mayoría mujeres; mayores, con edad media de 63 años, con prevalencia del $60 \%$ de sobrepeso y media de índice de masa corporal (IMC) promedio de $27,1 \mathrm{~kg} / \mathrm{m}^{2}$. Se ha encontrado fallas en el uso de fármacos antihipertensivos, debido a falta de supervisión periódica. Después de realizar intervenciones, el $86,7 \%$ de los pacientes presentaron reducción de los valores de presión. Los hallazgos apuntan para la importancia de un enfoque sistemático, continuo y seguimiento longitudinal de los pacientes y la importancia de los estudiantes de medicina ejercitaren prácticas en los servicios de APS en trabajo interprofesional. El estudio también ha demostrado la importancia de la APS como puerta de entrada de las personas en los servicios de salud, en la prevención de complicaciones de las enfermedades.

Palabras-claves: Educación Médica; Educación para la Salud; Hipertensión; Atención Primaria de Salud. 


\section{Introdução}

A evidência científica aponta para potenciais vantagens educacionais da inserção dos estudantes de Medicina em diferentes cenários de Atenção Primária à Saúde (APS) desde o início do curso. Mais especificamente, neste estudo abordaremos o Internato Rural Médico, como lócus privilegiado de cuidado centrado na pessoa, na família e na comunidade, com ênfase no trabalho interprofissional, em equipe, de forma horizontalizada, compartilhada, e tendo como foco as necessidades e desejos da pessoa sob cuidado, família e comunidade e a compreensão destes sobre o processo saúde, doença e adoecimento ${ }^{1,2}$. De entre as vantagens destacadas por educadores e estudantes, salienta-se: o avanço no engajamento dos estudantes com a comunidade, a melhoria do humanismo e da formação a partir das principais necessidades da população, o incentivo ao engajamento do trabalho em equipe interdisciplinar com responsabilidade e alteridade e a fixação dos médicos nas áreas mais carentes e remotas ${ }^{3,4,5}$.

Neste sentido, no ano de 2014, o Ministério da Educação do Brasil, por meio da Resolução $\mathrm{n}^{\mathrm{o}}$ 3, de 20 de junho de 2014, instituiu as Diretrizes Curriculares Nacionais (DCN) do Curso de Graduação em Medicina ${ }^{1}$, que em seu artigo $3^{\circ}$, determina que: o graduado em Medicina terá formação geral, humanista, crítica, reflexiva e ética, com capacidade para atuar nos diferentes níveis de atenção à saúde, com ações de promoção, prevenção, recuperação e reabilitação da saúde, nos âmbitos individual e coletivo, com responsabilidade social e compromisso com a defesa da cidadania, da dignidade humana, da saúde integral do ser humano e tendo como transversalidade em sua prática, sempre, a determinação social do processo de saúde e doença. A partir de então, é necessário que as instituições formadoras foquem a formação do graduado em Medicina tendo como eixo três áreas de atuação: Atenção à Saúde, Gestão em Saúde e Educação em Saúde, visando uma adequada articulação entre conhecimentos, habilidades e atitudes (competências) requeridas do egresso, para o futuro exercício profissional do médico ${ }^{1}$.

Segundo as DCN ${ }^{1}$, o estudante universitário deve ser capaz de realizar análise crítica e integradora da realidade e ter aptidão para socializar conhecimentos em vários contextos e públicos diferenciados. Além disso, salienta-se habilidades a serem desenvolvidas durante a formação profissional do aluno e temas, que transcendem ao ambiente próprio de sua formação, destacandose projetar ações de intervenção e propor soluções para situação-problema em cenários reais de aprendizagem.

Referente à atenção à saúde, a formação do estudante de Medicina, deve considerar o contexto social, político, cultural e ambiental dos sujeitos que necessitam de cuidados, tendo como eixos orientadores uma prática médica contínua e integrada visando à construção de projetos 
ISSN 2179-6750

terapêuticos compartilhados, estimulando o empoderamento, o autocuidado e a autonomia das pessoas, famílias, grupos e comunidades, que estimule o protagonismo dos usuários sobre sua própria saúde ${ }^{1,2,6,7}$. De forma complementar, mas não menos importante, a atenção à saúde deve estar pautada nas políticas públicas, programas, projetos, ações estratégicas e diretrizes atuais, visando o intervir qualificado no quadro de morbimortalidade da população loco-regional.

No Brasil, a Estratégia de Saúde da Família (ESF), objetiva reorganizar o Sistema Único de Saúde (SUS), a partir da mudança de um modelo centrado no hospital para um modelo cuja ênfase é a APS, com foco no trabalho em equipe interprofissional, a partir da estruturação do cuidado no território onde a vida das pessoas acontece - o mundo do trabalho e da vida cotidiana. Destarte, os princípios da integralidade, longitudinalidade, territorialidade e focalização na família - inscritos na ESF - fazem com que esta estratégia seja o modelo mais adequado para alterar o quadro das enfermidades prevenindo os agravos, além de promover a saúde ${ }^{2,6,7,8}$.

Mais especificamente, no que se refere ao quadro sanitário brasileiro, com o aumento da expectativa de vida, aumentou-se também a prevalência de doenças crônicas não transmissíveis (DCNT), sendo a hipertensão arterial (HA) a mais frequente delas. Caso não sejam adequadamente gerenciadas, as condições crônicas não só serão a primeira causa de incapacidade em todo o mundo até o ano 2020 como também se tornarão o problema mais dispendioso ${ }^{9,10}$. A HA é uma condição clínica multifatorial caracterizada por níveis elevados e sustentados de pressão arterial (PA). Associa-se frequentemente a alterações funcionais e/ou estruturais dos órgãos-alvo e a alterações metabólicas, com consequente aumento do risco de eventos cardiovasculares fatais e não fatais $10,11,12,13$

Trata-se uma doença altamente prevalente, de alto custo social e grande impacto na morbimortalidade da população mundial. Em estudos mundiais, a prevalência varia entre 22,3\% e 43,9\% (média de 32,5\%), sendo que 50\% destes estão entre 60 e 69 anos. Nas Américas, ela afeta 140 milhões de pessoas, entretanto $50 \%$ delas não sabem que são portadoras de HA ${ }^{10-15}$. No Brasil, a prevalência também é muito alta, são 17 milhões de hipertensos, de acordo com o critério atual de HA, que é PA maior ou igual a 140/90 mmHg. A Organização Mundial de Saúde (OMS) alertou que, para o ano de 2020, as mortes por DCNT representarão $73 \%$ dos óbitos no mundo ${ }^{9,12-14}$.

Uma das problemáticas da HA é o seu curso silencioso, o que implica no atraso do início do tratamento e nas baixas taxas de controle da doença. No entanto, 60 a $80 \%$ dos casos podem ser tratados em nível de APS, pois são de fácil diagnóstico, não requerem tecnologia sofisticada, podendo ser tratados e controlados com medicamentos de baixo custo e mudanças de estilo de vida, o que é comprovadamente eficaz e de fácil aplicabilidade em nível de APS ${ }^{12,14,16}$. 
ISSN 2179-6750

Neste contexto, destaca-se que a baixa adesão ao tratamento é, seguramente, o maior desafio a ser enfrentado pela APS, o que pode ser revertido por meio de uma boa relação do paciente com a equipe interdisciplinar de saúde, que juntos estabelecerão vínculos e buscarão formas adequadas e criativas de enfrentamento desta enfermidade ${ }^{12}$.

A adoção da ESF como política prioritária da APS no Brasil, por sua conformação e processo de trabalho, compreende as condições mais favoráveis de acesso às medidas multissetoriais e integrais de abordagem da $\mathrm{HA}^{17}$.

As ESF trabalham visando superar a atenção centrada no curativo em detrimento do cuidado preventivo, uma vez que ao trabalhar em todos os aspectos da saúde de forma dinâmica, na perspectiva de uma abordagem integral, autônoma e resolutiva podem modificar o processo de saúde e doença de um indivíduo, família e comunidade ${ }^{18}$.

Além disso, destaca-se a importância do fortalecimento do Sistema de Cadastramento e Acompanhamento de Hipertensos e Diabéticos (HIPERDIA), que é um programa de reorganização da atenção à HA e ao diabetes mellitus, por meio de um sistema de cadastramento e acompanhamento dos portadores desses agravos atendidos pelo SUS, objetivando a prevenção de agravos e enfermidades e promoção da saúde ${ }^{12}$.

Neste sentido, este estudo tem como objetivo apresentar o relato de experiência da atuação de acadêmicos de Medicina em trabalho interdisciplinar no controle da HA em estágio de Internato rural na ESF.

\section{Métodos}

O estudo foi desenvolvido no município de Catas Altas da Noruega, zona da mata mineira, com uma população predominantemente rural $(65,6 \%)^{19}$.

Segundo informações do Serviço de Saúde do município, na época do estudo, a prevalência de HA era de 20,2\%. Os estudantes de Medicina em trabalho com a equipe de saúde da ESF acompanharam 70 pacientes durante os meses de julho a dezembro de 2014. Para o estudo, incluíram-se os pacientes que foram previamente diagnosticados pela ESF do município como portador de $\mathrm{HA}(\mathrm{n}=15$ pacientes$)$.

A investigação baseou-se em três etapas complementares, a saber:

1. Estudo observacional - cujo objetivo foi o de conhecer o território e as necessidades locais;

2. Palestras dialogadas para os portadores de HA - sobre temas importantes para o controle da HA, inicialmente delineados pela equipe de saúde e posteriormente a partir da 127 
ISSN 2179-6750

demanda dos pacientes;

3. Consultas individualizadas - a partir de um olhar crítico e escuta ativa e qualificada dos estudantes de Medicina e equipe de saúde, respeitando a singularidade de cada pessoa.

Na primeira etapa observacional, evidenciou-se que os portadores de HA não realizavam o devido acompanhamento médico, principalmente pelo fato do município não contar com um programa de saúde voltado para os portadores de HA. Esta deficiência no cuidado à saúde dos usuários da ESF acarretou em falta de adesão ao tratamento e descontrole da HA.

Após o levantamento dos dados, foram convidados todos os portadores de HA de 7 regiões específicas da cidade para participarem de atividades em grupos, onde os estudantes de Medicina e o enfermeiro da ESF, proferiram palestras dialogadas sobre orientações gerais da doença e hábitos de vida saudáveis; além de tratarem também de temas apontados como importantes pelos portadores de HA.

O terceiro passo, complementar e sequencial, foi o agendamento de consultas individualizadas com os participantes do estudo, introduzindo concomitantemente, na ESF, as ações do programa HIPERDIA. Para tal, utilizou-se um formulário padronizado do próprio programa, com as seguintes informações: sexo, idade, história de outras doenças, uso de medicamentos, peso, altura e valores da PA, hábitos de vida e vícios como tabagismo e etilismo. Objetivou-se estabelecer um acompanhamento individualizado conforme as necessidades de tratamento de cada paciente. Este acompanhamento permitiu uma adequação dos medicamentos anti-hipertensivos, aqueles que não estavam adequados, tanto em sua posologia quanto em sua ação terapêutica foram alterados.

Posteriormente, foi marcada uma segunda consulta, na qual foi avaliada a eficácia das intervenções realizadas e posteriores consultas caso fosse necessário.

O presente estudo faz parte de um projeto maior do Programa de Inovação em Docência Universitária (PRODUS) e do Laboratório de Planejamento e Gestão em Saúde (LabPlanGest), e foi aprovado pelo Comitê de Ética em Pesquisa da Universidade Federal de Viçosa (Protocolo $\mathrm{n}^{\mathrm{o}}$ 091/2010). De acordo com a resolução $n^{\circ}$ 466/2012 do Conselho Nacional de Saúde, que regulamenta as pesquisas envolvendo seres humanos, foi solicitado consentimento livre e esclarecido dos indivíduos para participarem do estudo, garantindo-se a confidencialidade das informações e o anonimato dos mesmos.

\section{Resultados}

Participaram deste estudo, 15 portadores de HA, sendo a maioria do sexo feminino $(66,7 \%)$; idosos, com média de idade de 63 anos, mínima de 50 e máxima de 86 anos; não tabagistas (80\%) e 


\section{ISSN 2179-6750}

não etilistas (100\%). Em relação ao estado nutricional, encontrou-se uma prevalência de $60 \%$ de sobrepeso, com média de índice de massa corporal (IMC) de $27,1 \mathrm{~kg} / \mathrm{m}^{2}$. As demais características da população se encontram na Tabela 1.

Tabela 1. Caracterização da população estudada. Catas Altas da Noruega-MG, 2014.

\begin{tabular}{|c|l|c|c|}
\hline \multicolumn{2}{|c|}{ Variáveis } & $n$ & Prevalência (\%) \\
\cline { 2 - 4 } & & & \\
\hline \multirow{2}{*}{ Sexo } & Masculino & 5 & 33,3 \\
\cline { 2 - 4 } & Feminino & 10 & 66,7 \\
\hline \multirow{2}{*}{$\begin{array}{c}\text { Idade } \\
\text { (anos) }\end{array}$} & $25-50$ & 1 & 6,6 \\
\cline { 2 - 4 } & $51-60$ & 6 & 40,0 \\
\cline { 2 - 4 } & $61-70$ & 4 & 26,7 \\
\cline { 2 - 4 } & $\geq 71$ & 4 & 26,7 \\
\hline \multirow{2}{*}{ Uso de álcool } & Não & 15 & 100,0 \\
\cline { 2 - 4 } & Sim & 3 & 0,0 \\
\hline \multirow{2}{*}{ Tabagista } & Não & 6 & 20,0 \\
\cline { 2 - 4 } & Sim & 9 & 40,0 \\
\hline Excesso de peso & Não & & 60,0 \\
\cline { 2 - 4 } & Sim & & \\
\hline \multirow{2}{*}{ Fonte: próprios autores } & & & \\
\hline
\end{tabular}

Em relação às estratégias de controle da HA realizadas com a população, destacam-se o desenvolvimento de palestras dialogadas e dinâmicas, além de visitas domiciliares e acompanhamento dos portadores de HA por meio de consultas individualizadas. Obteve-se um resultado bastante positivo, com redução média de $13,8 \mathrm{mmHg}$ nos valores de PA aferidos antes e após as intervenções. Destaca-se que 86,7\% (n=13) dos participantes do estudo tiveram redução nos valores da PA, conforme se visualiza no Gráfico 1.

Em relação ao uso de medicamentos anti-hipertensivos, encontraram-se diversas falhas, principalmente devido à falta de acompanhamento periódico e sistematizado pelos profissionais de saúde da ESF.

De entre os principais problemas relacionados ao uso de medicamentos destacam-se a dosagem e/ou tipos de medicamentos inadequados. Cita-se, por exemplo, que os pacientes em uso de Metildopa (simpaticolítico de ação central), medicamento principalmente indicado para doentes renais crônicos e gestantes, não se encontravam em nenhuma destas duas condições; pacientes em uso de Captopril (inibidores da enzima conversora da angiotensina - IECA), que tomavam um comprido ao dia, sendo que deveria ser utilizado no mínimo um comprimido a cada 12 horas, devido a sua meia vida curta. 
Gráfico 1. Valores de PA antes e após o desenvolvimento de estratégias de controle da HA. Catas Altas da Noruega-MG, 2014.

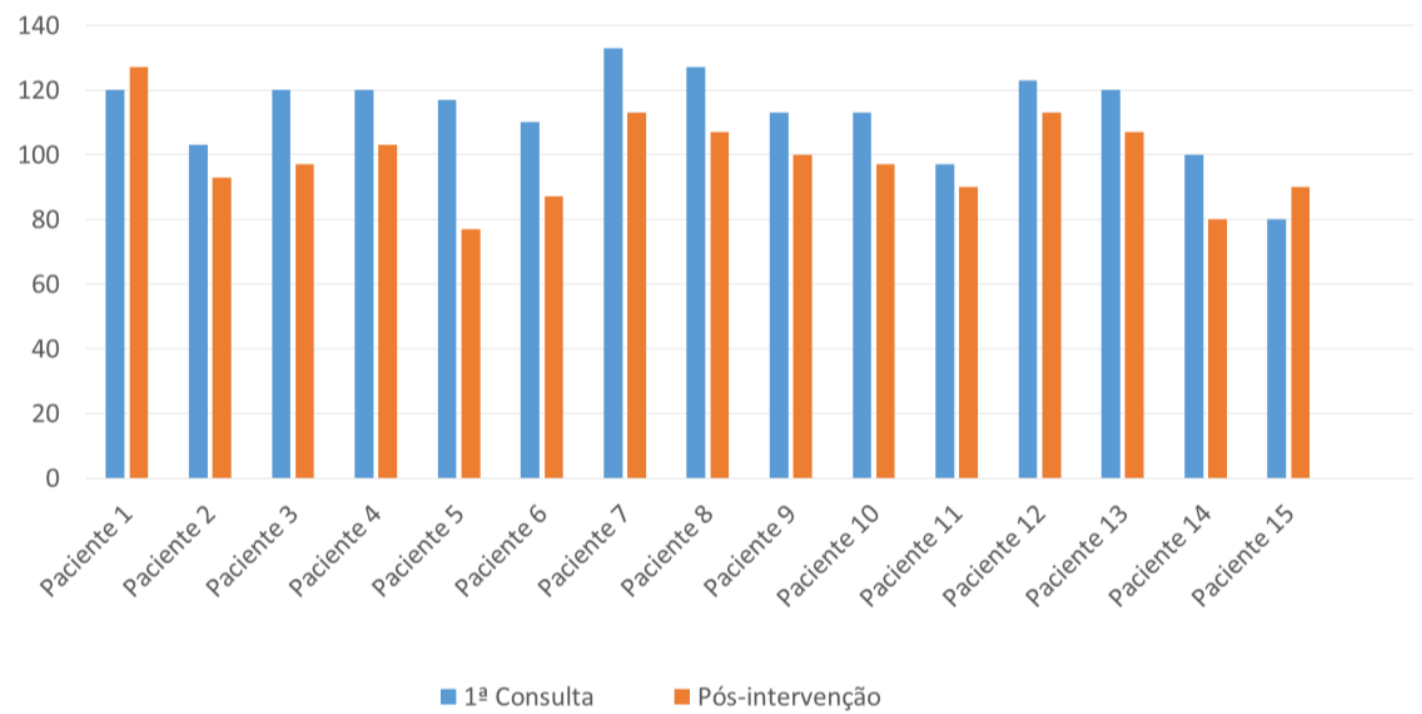

Neste contexto, é importante salientar o papel estratégico do Internato Médico em cenários da APS, mais enfaticamente na ESF, enquanto possibilidade de vivência prática e inovadora para os estudantes de Medicina, bem como, para a equipe de saúde. Mais especificamente, a vivência tanto nas unidades de saúde (atendimentos individuais e trabalho com grupos de usuários por equipe interprofissional), como nas visitas domiciliares contribuiu para a formação de futuros médicos comprometidos com o trabalho em equipe e com a APS da forma como foi preconizada pelo Movimento da Reforma Sanitária Brasileira. Destaca-se ainda, o entendimento por parte dos estudantes sobre o SUS em cenários reais de prática, propiciando o comprometimento com a política de saúde brasileira. Assim, a experiência do Internato Rural, aqui relatada, trouxe para os estudantes de Medicina, enquanto membros de uma equipe interprofissional, a certeza de que apesar dos problemas inerentes a implementação do SUS nos municípios, esta é a mais adequada política social em curso no Brasil.

\section{Discussão}

A HA é uma doença crônica de alta prevalência, representando um grave problema de saúde pública no Brasil e no mundo e um fator de risco independente para doenças cardiovasculares e renais. De acordo com o Protocolo de Hipertensão Arterial Sistêmica para a APS ${ }^{20}$, a HA é o problema de saúde mais frequente de consulta médica nas unidades de saúde, representando 17\% 
ISSN 2179-6750

das consultas realizadas.

Estudos americanos apontam que até os 40 anos a prevalência é próxima a 10\% (20\% para a raça negra), até os 50 anos chega a 20\% (40\% para a raça negra), após 60 anos ultrapassa os 40\%, atingindo $60 \%$ após 70 anos. Estudos mais recentes com a população brasileira demonstram uma prevalência de $26 \%$ da população geral adulta, e aproximadamente $50 \%$ da população idosa, variando de acordo com a localidade ${ }^{10,21-23}$. De forma semelhante, no presente estudo, encontrou-se que mais de $50 \%$ dos portadores de HA avaliados eram idosos.

Em relação ao gênero, a maioria dos indivíduos avaliados era mulheres (66,7\%). Estudos presentes na literatura mostram que apesar de as taxas masculinas assumirem um peso significativo nos perfis de morbimortalidade, observa-se que a presença de homens nos serviços de APS é menor do que a das mulheres ${ }^{24,25}$, o que vai ao encontro dos achados do presente estudo, que evidenciou uma maior prevalência de mulheres com HA

Quanto ao estado nutricional, diferentes estudos evidenciam o sobrepeso como fator de risco para desenvolvimento e agravamento da $\mathrm{HA}^{13,22,23}$, o que também foi encontrado neste estudo, já que $60 \%$ dos avaliados apresentavam sobrepeso.

Os achados do presente trabalho apontam ainda para a importância de um acompanhamento sistemático, contínuo e longitudinal dos portadores de HA, caraterísticas estas que devem ser colocadas em prática pelas equipes da ESF. Neste sentido, destaca-se a importância de que os estudantes de Medicina tenham a possibilidade de trabalhar com equipe interprofissional durante sua formação acadêmica no acompanhamento e tratamento tanto individualizado como em grupos, em consultas clínicas e em visitas domiciliares.

A maioria dos portadores de HA participantes do estudo apresentava a PA descompensada por estarem em uso de medicamentos e/ou dosagens inadequados, o que pode ser devido à falta de acompanhamento periódico conforme recomendado pelas VI Diretrizes Brasileiras de Hipertensão Arterial ${ }^{13}$.

Enfatiza-se que o controle da HA está diretamente relacionado ao grau de adesão do portador ao tratamento. No entanto, estima-se que apenas um terço das pessoas acompanhadas em serviços de saúde tem sua PA mantida em níveis desejáveis, o que pode ser explicado pela baixa adesão ao tratamento. Estudos apontam controle dos níveis pressóricos entre $20 \%$ a $40 \%$ e acrescentam que a taxa de abandono, grau mais elevado da falta de adesão, cresce com o tempo de terapêutica. Além disso, cerca de $40 \%$ a $60 \%$ dos portadores de HAS não fazem uso da medicação prescrita, o que reforça a importância do acompanhamento longitudinal dos pacientes ${ }^{12,22}$.

No Brasil, a ESF tem importância primordial nas ações relacionadas à APS. As práticas da 131 
ISSN 2179-6750

ESF têm como eixo central o trabalho com indivíduos, famílias e comunidades em um contexto territorial específico, estimulando assim o vínculo e o trabalho contínuo, executado por uma equipe multiprofissional e interdisciplinar. $O$ foco deixa de ser apenas uma intervenção centrada na consulta médica, ampliando a abordagem ao estimular além do trabalho individualizado, intervenções e orientações em equipe a diferentes grupos populacionais. Além da abordagem multiprofissional e em grupo destaca-se também a assistência domiciliar, o que permite uma maior inserção e conhecimento da realidade de vida da população e de suas necessidades, facilitando assim o estabelecimento de vínculos e relações de confiança entre os profissionais e os pacientes 8,22 .

Estudos presentes na literatura apontam que a equipe da ESF, deve desenvolver estratégias de educação e promoção da saúde. Segundo Ribeiro et al ${ }^{22}$ "a disseminação da informação e a educação em saúde são fundamentais para a tomada de decisão e, por isso, componentes importantes da promoção da saúde, por estarem ligadas ao princípio de empoderamento e libertação de pessoas, famílias e coletividades”. Além da disponibilização e acesso de informações, o paciente portador de HA necessita ser acompanhado sistematicamente e por um período de tempo contínuo e longitudinal, tendo na equipe interprofissional um apoio para a tomada de decisões sobre o seu viver cotidiano e sua relação com a enfermidade e não raras vezes com as possíveis complicações que esta impõe ${ }^{22,8,26}$.

Destaca-se ainda a importância da implantação do HIPERDIA pela equipe da ESF no município, o que permitiu o cadastramento e acompanhamento destes pacientes, propiciando assim, o desenvolvimento de ações visando o controle da HA na própria ESF, por meio de ações individuais e coletivas.

Referente às atividades em grupo, a equipe médica e de enfermagem, realizou palestras dialogadas e dinâmicas com os portadores de HA, versando sobre orientações relativas aos hábitos de vida saudáveis e à importância do uso de medicamentos na dosagem correta segundo indicação de cada paciente. Estas atividades propiciaram a formação de vínculos entre os profissionais de saúde e os pacientes e familiares, além da redução dos valores de PA e consequente melhoria da qualidade de vida da população em estudo.

Sendo assim, as orientações em grupo de forma dialógica e o acompanhamento dos pacientes foram fundamentais para o estabelecimento do vínculo entre os profissionais de saúde e os pacientes, o que refletiu nas mudanças de hábitos e melhoria da qualidade de vida. Além disso, por meio das consultas individualizadas foi possível traçar um acompanhamento terapêutico específico conforme as peculiaridades de cada paciente, alterando os medicamentos e/ou as 
ISSN 2179-6750

dosagens sempre que necessário.

O estudo demonstra ainda a importância da APS, visto que esta é a porta de entrada dos indivíduos nos serviços de saúde, e o lugar onde o controle e a prevenção de complicações devem ser estabelecidos. Há que se salientar ainda, o papel primordial da ESF em municípios de pequeno porte, já que esta constitui a única possibilidade de cuidado terapêutico em nível local.

\section{Considerações Finais}

Os achados deste estudo apontam para os benefícios educacionais da inserção dos estudantes de Medicina na ESF, por meio do Internato Rural, tendo na comunidade um lócus privilegiado de aprendizado e transformação significativa do processo de ensino e aprendizagem, ao mesmo tempo em que gera benefícios para a equipe e a população. Isto foi vivenciado tanto nas atividades individuais realizadas pelos estudantes de Medicina, quanto nos diferentes trabalhos em equipe interprofissional, destacando-se as atividades em grupos e visitas domiciliares. Nestes cenários, o contato com os usuários propiciou a compreensão dos princípios do SUS de forma prática e contextualizada.

\section{Referências}

1. Brasil. Ministério da Educação. Conselho Nacional de Educação. Câmara de Educação Superior. Resolução n 3, de 20 de junho de 2014. Institui Diretrizes Curriculares Nacionais do Curso de Graduação em Medicina e dá outras providências. Brasília; 2014.

2. Cotta RMM, Campos AAO, Mendonça ET, Costa GD, Machado JC, Silva LS, et al. Políticas de Saúde: desenhos, modelos e paradigmas. Viçosa: Editora UFV/ABRASCO; 2013.

3. Strasser R, Hirsh D. Longitudinal integrated clerkships: transforming medical education worldwide? Med Educ. 2011; 45(5):436-7.

4. Walters L, Prideaux D, Worley P, Greenhill J. Demonstrating the value of longitudinal integrated placements to general practice preceptors. Med Educ. 2011; 45(5):455-63.

5. Mazotti L, O’Brien B, Tong L, Hauer KE. Perceptions of evaluation in longitudinal versus traditional clerkships. Med Educ. 2011; 45(5):464-70.

6. Cotta RMM, Reis RS, Batista KCS, Costa GD, Alfenas RCG, Castro FAF. Hábitos e práticas alimentares de hipertensos e diabéticos: repensando o cuidado a partir da atenção primária. Rev de Nutr, 2009; 22(6):823-835.

7. Ribeiro AG, Cotta RMM, Ribeiro SMR, Dias CMGC, Araújo RMA. Representações sociais 133 
ISSN 2179-6750

de mulheres portadoras de hipertensão arterial sobre sua enfermidade: desatando os nós da lacuna da adesão ao tratamento na agenda da Saúde da Família. Physis. 2011; 21(1):87-112.

8. Cotta RMM, Reis RS, Carvalho AL, Siqueira KC, Castro FAF, Alfenas RCG. Reflexões sobre o conhecimento dos usuários no contexto do Programa de Saúde da Família: a lacuna entre o saber técnico e o popular. Physis. 2008; 18(4):745-66.

9. Silva LS, Cotta RMM, Rosa COB. Estratégias de promoção da saúde e prevenção primária para enfrentamento das doenças crônicas: revisão sistemática. Rev Panam Salud Publica. 2013; 34(5):343-50.

10. Teixeira JBP, Eiras NSV. A Hipertensão Arterial e sua abordagem pela Atenção Primária à Saúde e pelos Grupos de Extensão Universitária. s/d. [online]. [acessado em 15 Dez 2014] Disponível em: http://www.ufjf.br/proplamed/files/2011/04/A-Hipertens\%C3\%A3oArterial-e-sua-abordagem-pela-APS.pdf.

11. Silva AMN, Nunes JFC, Quintas RN, Moura TBC. Epidemiologia dos pacientes com hipertensão arterial cadastrados na "Casa Família Água Cristal", Belém-PA. Rev. Para. Med. 2007; 21(1): 47-47.

12. Cunha CW. Dificuldades no controle da hipertensão arterial sistêmica e diabetes mellitus na atenção básica de saúde através do Hiperdia - Plano de Reorganização da Atenção. (Trabalho de Conclusão de Curso). Universidade Federal do Rio Grande do Sul; 2009.

13. Sociedade Brasileira de Cardiologia / Sociedade Brasileira de Hipertensão / Sociedade Brasileira de Nefrologia. VI Diretrizes Brasileiras de Hipertensão (VI DBHA). Arq Bras Cardiol. 2010; 95(1 supl.1): 1-51.

14. Sousa LB, Souza RKT, Scochi MJ. Hipertensão arterial e saúde da família: atenção aos portadores em município de pequeno porte na região Sul do Brasil. Arq. Bras. Cardiol. 2006; 87(4).

15. Sala A, Nemes-Filho A, Eluf-Neto J. Avaliação da efetividade do controle da hipertensão arterial em unidade básica de saúde. Rev. Saúde Pública. 1996; 30(2).

16. Brasil. Conselho Nacional de Secretários de Saúde. Atenção Primária e Promoção da Saúde / Conselho Nacional de Secretários de Saúde. - Brasília: CONASS; 2007.

17. Brasil. Ministério da Saúde. Secretaria de Vigilância em Saúde. Departamento de Análise de Situação de Saúde. Plano de ações estratégicas para o enfrentamento das doenças crônicas não transmissíveis (DCNT) no Brasil 2011-2022 / Ministério da Saúde. Secretaria de Vigilância em Saúde. Departamento de Análise de Situação de Saúde. - Brasília: Ministério da Saúde; 2011. 
ISSN 2179-6750

18. Famema - Faculdade de Medicina de Marília. Secretaria de Ensino Superior. Secretaria Municipal de Saúde de Marília. Educação em saúde com usuários hipertensos: integralidade no cuidado. Marília; 2009.

19. IBGE - Instituto Brasileiro de Geografia e Estatística. Censo 2010. [acessado em: 01 de nov de 2014]. Disponível em: http://censo2010.ibge.gov.br/.

20. Brasil. Ministério da Saúde. Grupo Hospitalar Conceição. Protocolo de Hipertensão Arterial Sistêmica para a Atenção Primária em Saúde; organização de Sandra Rejane Soares Ferreira. et.al; ilustrações de Maria Lúcia Lenz. Porto Alegre: Hospital Nossa Senhora da Conceição, $2009.54 \mathrm{p}$.

21. Cavagioni LC, Pierin AMG. Hipertensão arterial em profissionais que atuam em serviços de atendimento pré-hospitalar. Texto contexto - enferm. 2011; 20(3): 435-444.

22. Ribeiro AG, Cotta RMM, Silva LS, Ribeiro SMR, Dias CMGC, Mitre SM, et al. Hipertensão arterial e orientação domiciliar: o papel estratégico da saúde da família. Rev. Nutr. 2012; 25(2): 271-282.

23. Carvalho MV, Siqueira LB, Sousa ALL, Jardim PCBV. The influence of hypertension on quality of life. Arq. Bras. Cardiol. 2013; 100(2):164-174.

24. Pinheiro RS, Viacava F, Travassos C, Brito AS. Gênero, morbidade, acesso e utilização de serviços de saúde no Brasil. Ciênc Saúde Coletiva. 2002; 7:687-707.

25. Figueiredo W. Assistência à saúde dos homens: um desafio para os serviços de atenção primária. Ciênc Saúde Coletiva. 2005; 10:105-109.

26. Brasil. Ministério da Saúde. Secretaria de Políticas de Saúde. As cartas da promoção da saúde. Brasília: MS; 2002. 